\title{
Diagnosing chronic rhinosinusitis: comparing questionnaire-based and clinical-based diagnosis*
}

\author{
B. Lange' ${ }^{1}$ T. Thilsing ${ }^{2}$, J. Baelum² ${ }^{2}$ R. Holst ${ }^{3}$, A. Kjeldsen \\ ' Department of Otorhinolaryngology, Odense University Hospital, University of Southern Denmark, Odense, Denmark \\ 2 Department of Occupational and Environmental Medicine, Odense University Hospital, Odense, Denmark \\ ${ }^{3}$ Department of Biostatistics, University of Southern Denmark, Odense, Denmark
}

Rhinology 51: 128-136, 2013

DOl:10.4193/Rhino12.029

*Received for publication:

February 8, 2012

Accepted: February 21, 2013

\section{Summary}

Background: The European Position Paper on Rhinosinusitis and Nasal Polyps (EP3 OS) incorporates symptomatic and endoscopic criteria in the clinical diagnosis of chronic rhinosinusitis (CRS), while in epidemiological studies the definition is based on symptoms only. The aim of this study was to evaluate the correlation between questionnaire-based and clinical-based CRS.

Methods: Based on the GA²LN postal survey data a total of 366 persons participated at the follow-up at the Danish centre and provided information on questionnaire-based CRS. At the same occasion the 366 participants underwent clinical interview and examination by an otorhinolaryngologist to provide information for a clinical-based CRS diagnosis. The association between questionnaire-based and clinical-based CRS diagnosis was determined using logistic regression models and kappa statistics.

Results: Mean age of respondents to the postal questionnaire was 45.3 years and $52.9 \%$ were female. Persons with asthma were $8.4 \%$ and $26.2 \%$ were actual smokers. There was moderate agreement between questionnaire-based and clinicalbased CRS. Sensitivity was low comparing questionnaire-based CRS with clinical-based CRS. Incorporation of self reported CRS and medical history in diagnosing CRS by questionnaire increased the agreement and sensitivity while specificity stayed at a high level.

Conclusion: Evaluating the correlation between questionnaire-based and clinical-based CRS showed only moderate agreement and questions whether they evaluate the same disease. It brings into consideration that adjustments are needed to justify correlation between questionnaire-based and clinical-based diagnosis of CRS.

Key words: chronic rhinosinusitis, questionnaires, Sino Nasal Outcome Test 22

\section{Introduction}

Chronic rhinosinusitis (CRS) is a common disease with considerable impact on quality of life and airway morbidity. Recently, the first trans-European study on CRS was conducted and the overall prevalence of self reported CRS prevalence was $10.9 \%{ }^{(1)}$. The prevalence of CRS is higher among smokers, persons reporting asthma, and manual workers ${ }^{(2)}$. Until now, valid epidemiological studies have been difficult to conduct due to lack 
of generally accepted criteria and definition on CRS. In 2007, the $\mathrm{EP}^{3} \mathrm{OS}$ document, initiated by the Academy of Allergology and Clinical Immunology (EAACI) and approved by the European Rhinology Society (ERS), was introduced and it defines CRS both in relation to epidemiology, research, and practitioners ${ }^{(3)}$. The $\mathrm{EP}^{3} \mathrm{OS}$ document gives consensus on the criteria for epidemiological studies being based on specific self reported symptoms and for clinical studies the symptoms are combined with results of clinical examination and/or a CT scan (Table 1). The clinical diagnosis is made by a doctor, preferably an otolaryngologist, who performs a clinical interview and an endoscopy of the nasal cavity. In population based large scale studies endoscopy and also CT-scan may unfortunately not be possible and therefore symptom-based questionnaires for CRS have been developed. A CT-scan has been proposed as part of the clinical diagnosis. However, it is not suitable for wide scale use in epidemiological studies and although some have found that it correlates well with endoscopic scores ${ }^{(4)}$ the correlation between $\mathrm{CT}$ findings and symptom scores has generally been shown to be poor ${ }^{(5,6)}$.

In 2011, as part of GA²LEN, Tomassen et al. found that a questionnaire-based definition of CRS had a moderate reliability over time, and they found that there was a significant association between questionnaire-based CRS and positive endoscopy ${ }^{(7)}$. In their study the CRS diagnosis was based on the survey questionnaire and the results of endoscopy was an isolated observation not included in the diagnosis.

Concerning CRS the EP ${ }^{3} \mathrm{OS}$ document has tried to accommodate different needs by epidemiologist, researchers, and practitioners by offering different definitions that can be applied under different circumstances.

One of the issues of epidemiological research is whether the chosen questionnaire identifies a disease with the same accuracy as the clinician. If this is not the case, we cannot be sure that the disease diagnosed by the questionnaire is the same as the disease diagnosed by the clinician.

Evaluation of questionnaire-based diagnosis compared to clinical-based diagnosis is important to make the epidemiological instruments stronger. There seems to be a lack of such studies in diagnosing CRS.

The aim of the present study was, at the same occasion, to compare the questionnaire-based diagnoses of CRS with the clinicalbased diagnosis of CRS in a population-based sample.

\section{Material and methods}

\section{Survey}

As the Danish participant in a trans-European based GA2LEN project, a postal questionnaire was send to a representative random sample of 5,000 subjects (2,500 women and 2,500 men) aged 15-75 years. All subjects were living on the Danish island Funen. The questionnaire was newly developed for the diagnosis of CRS and a positive diagnosis of CRS was based on symptoms as defined in the $2007 \mathrm{EP}^{3} \mathrm{OS}$ epidemiological criteria $^{(3)}$ (Table 1). In addition, a question on self reported doctor diagnosed CRS was included: "Has a doctor ever told you that you have chronic sinusitis?".

For the diagnosis of asthma and allergic rhinitis, ECRHS (European Community Respiratory Health Survey) questions were used. Asthma was defined as an affirmative answer to the question: "Have you ever had asthma?" and a positive answer to at least one of the following symptoms in the last 12 month: (i)

Table 1. Title. Definition of symptom-based and clinical-based diagnosis of CRS.

Symptom-based definition of chronic rhinosinusitis defined by $\mathrm{EP}^{3} \mathrm{OS}$

Presence of two or more of the following symptoms

- Nasal blockage/obstruction/congestion

Nasal discharge (anterior/posterior nasal drip)

Facial pain/pressure

Reduction or loss of smell

One of which should be blockage or discharge

Duration of symptoms $>12$ weeks

Clinical-based definition of chronic rhinosinusitis defined by an otolaryngologist using 1. (EP ${ }^{3} \mathrm{OS}$ ) or 2. (known $\mathrm{CRS}+$ relevant treatment)

1.

Presence of two or more of the following symptoms at interview:

- Nasal blockage/obstruction/congestion

- Nasal discharge (anterior/posterior nasal drip)

- Facial pain/pressure

- Reduction or loss of smell

And

Rhinoscopic signs of

-Polyps:

o $0=$ none

o $1=$ polyps not reaching the lower edge of the middle turbinate

- 2 = polyps beyond middle meatus (but not obstructing the nasal cavity)

o $3=$ polyps completely obstructing the nasal cavity

And/Or

-Mucopurulent discharge, primarily from middle meatus, and/or

o $0=$ none

o 1 = mucous

o 2 = purulent

And/Or

- Oedema or obstruction primarily in middle meatus

o $0=$ none

o $1=$ mild

o 2 = moderate

o 3 = severe

Or

2.

Medical history of CRS

And

Relevant medical treatment of CRS 
wheeze or whistling in the chest, (ii) waking with chest tightness, (iii) waking with shortness of breath, and (iv) waking with an attack of coughing ${ }^{(8)}$. Allergic rhinitis was defined by an affirmative answer to the question "Do you have any nasal allergies including hay fever?"

Patients with chronic rhinitis have symptoms resembling those of CRS. Chronic rhinitis was in this study defined as having nasal symptoms for more than 12 weeks without fulfilling the $\mathrm{EP}^{3} \mathrm{OS}$ criteria for CRS.

Current smokers were defined by an affirmative answer to the question "Have you smoked at all in the last month?". Former smokers were defined as participants answering yes to the question "Have you ever smoked for as long as a year?" (Yes means at least one cigarette per day or one cigar per week for one year) and no to the question "Have you smoked at all in the last month?".

\section{Follow-up}

In a second phase (the GA2LEN survey follow-up), selected respondents to the questionnaire were invited to a clinical examination. According to the protocol, the aim was to include 120 subjects with asthma, 120 with CRS, 40 with asthma and CRS, and 120 with neither asthma nor CRS according to the above mentioned criteria. At the follow-up, the participants were interviewed by a clinical investigator including repetition of the same questionnaire as described for the postal survey. The questions for assessing CRS by questionnaire are shown in Table 2 and the questionnaire at follow-up was in this study used for the questionnaire-based CRS diagnosis. After fulfilling the questionnaire, the participants were interviewed and examined by a trained otolaryngologist. In the interview, the otolaryngologist included questions on CRS symptoms according to $\mathrm{EP}^{3} \mathrm{OS}$. The meeting with the otolaryngologist was build as in a normal clinical setting. For posterior rhinoscopy a flexible laryngoscope was used. Anterior rhinoscopy was performed before and after decongestion whereas posterior rhinoscopy was only performed after decongestion. A positive rhinoscopy was defined as presence of polyps, presence of oedema in the middle meatus,

Table 2. Questions for assessing CRS as per $\mathrm{EP}^{3} \mathrm{OS}$.

1. Has your nose been blocked for more than 12 weeks during the last 12 months?

2. Have you had pain or pressure around the forehead, nose, or eyes for more than 12 weeks during the last 12 months?

3. Have you had discoloured nasal discharge or discoloured mucus in the throat for more than 12 weeks during the last 12 months?

4. Has your sense of smell been reduced or absent for more than 12 weeks during the last 12 months? or presence of thick purulent discharge in the middle meatus at either nasal side. The otolaryngologist was blinded to the results of the questionnaires.

A positive diagnosis of CRS by the otolaryngologist was based on the $\mathrm{EP}^{3} \mathrm{OS}$ clinical criteria meaning the patient's symptoms on CRS together with findings by endoscopy. The clinical criteria are shown in Table 1 together with the rhinoscopic grading system. In addition, CRS was diagnosed if a patient, according to the interview, in his or her medical history, was known with CRS and was in current relevant medical treatment with nasal steroid. This additional assessment was regardless of reported symptoms and objective findings as a person with CRS in optimal treatment may have full relive of symptoms (control of disease).

A CT scan was not an option in this study due to ethical and economical reasons.

The patient filled out the Sino Nasal Outcome Test 22 (SNOT-22), which is a patient-reported measure of symptom severity and health-related QoL in sinonasal conditions recently validated for Danish patients ${ }^{(9)}$. It was used by the otolaryngologist as part of the structured interview focusing on the rhinological questions and was used to evaluate the severity of disease. The total sum score was divided into mild: 0-9, moderate: $10-29$, and severe: 30-110.

\section{The new definition}

In the evaluation of questionnaire-based CRS, the analysis was performed both with the original definition by $\mathrm{EP}^{3} \mathrm{OS}$ and with a new definition: EPOS based including Medical treated and Doctor diagnosed - CRS (EMD-CRS). In the new definition, we added self reported doctor diagnosed CRS and being in current treatment for CRS as an option for questionnaire-based CRS diagnosis as shown in Table 1. This option was regardless of the reported symptoms as a patient with known CRS and being in optimal medication may have relive of symptoms as noted above.

"The golden standard" of diagnosing CRS in this study was the clinical diagnosis of CRS performed by an otolaryngologist including nasal rhinoscopy.

\section{Statistical analysis}

Comparison of questionnaire-based CRS with clinical-based CRS was estimated using Cohens's kappa statistics which is an expression of strength of agreement.

The odds for the four possible combinations of agreement between questionnaire-based and clinical-based CRS (true positive, false positive, true negative, and false negative using the clinical-based diagnosis as the true reference) were estimated 
Sensitivity and Specificity Female

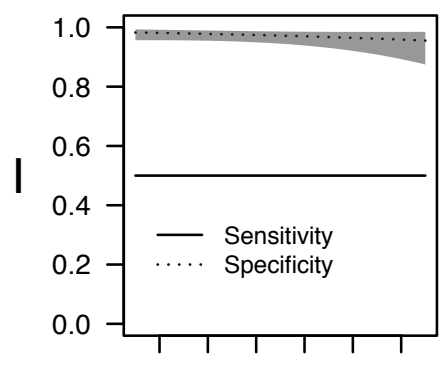

$20 \quad 40 \quad 60$

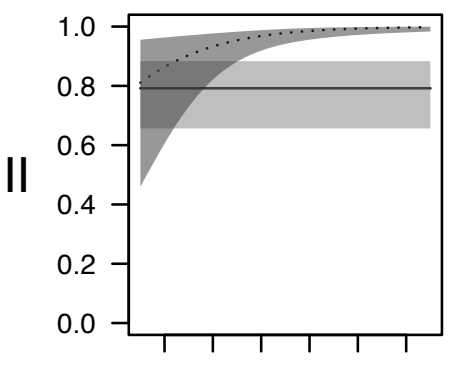

$20 \quad 40 \quad 60$
Male

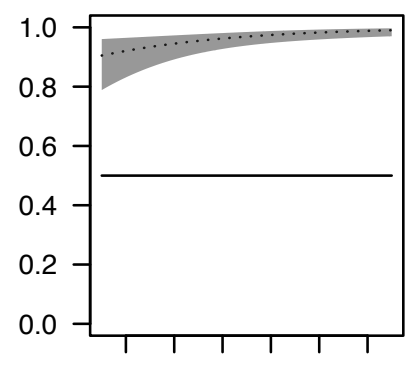

$20 \quad 40 \quad 60$

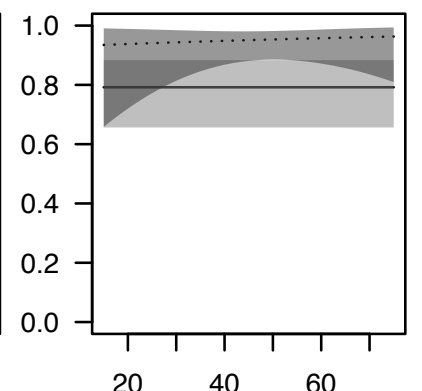

PPV and NPV

Female

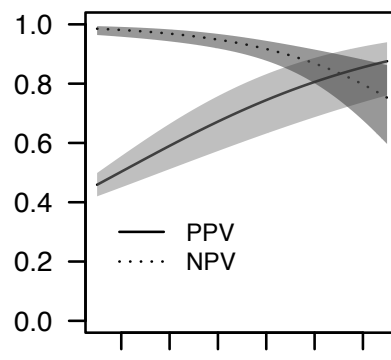

$20 \quad 40 \quad 60$

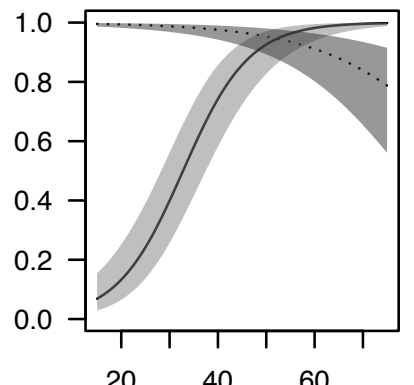

Male

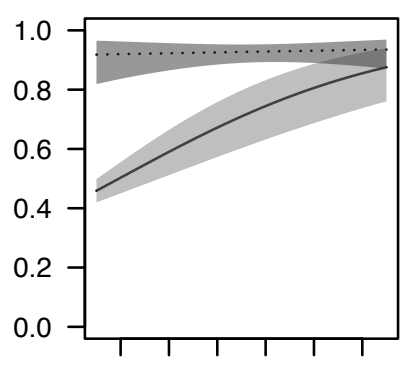

$20 \quad 40 \quad 60$

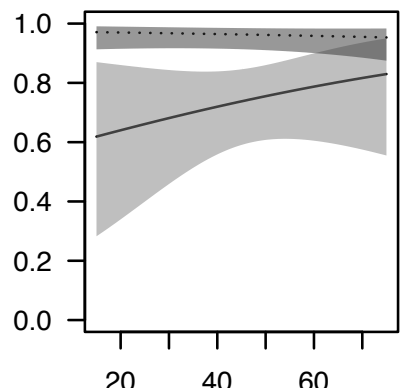

Age (years)

Figure 1. Title. Sensitivities, specificities, PPV, and NPV with 95\% confidence bands for patients with no asthma and no allergic rhinitis.

Row I: Questionnaire-based CRS compared to clinical-based CRS. Row II: EMD-CRS compared to clinical-based CRS.

by means of a multinomial logistic regression ${ }^{(10)}$. Two different models were considered. The first included only sex and age as covariates whereas the second also included asthma, allergic rhinitis, and smoking status. These estimated odds were subsequently used for calculating expected sensitivities, specificities, positive predictive (PPV), and negative predicted values (NPV) (11).

\section{Results}

Between May and August 2008, a total of 3,397 persons completed and returned the postal questionnaire with valid data (response rate $=67.4 \%$ ). Among the respondents, a total of 366 persons participated at the follow-up from October 2008 to March 2009. Mean age of the participants at follow-up was 47.9 and $54.2 \%$ were female. Based on sampling proportion, the prevalence of clinical-based CRS was estimated to $8.5 \%$ and the prevalence of questionnaire-based CRS was estimated to $7.0 \%$. In patients having clinical-based CRS, the average SNOT-22 total sum score was 28.12 and it showed that 12 patients (13.2\%) had mild symptoms, 44 (48.3\%) had moderate symptoms, and 35 (38.5\%) had severe symptoms.

According to the otolaryngologist, 91 persons had CRS and 271 persons had no CRS. Data on 4 persons were lost due to random failure during data administration.

Table 3 shows the distribution of persons who were diagnosed with CRS according to the questionnaire and according to the otolaryngologist. Only 45 cases were given both a questionnaire-based and clinical-based CRS diagnosis. The mean SNOT 22 total sum score in this group was 30.7. In 19 cases, CRS was diag-

Table 3. Overview of number of persons with or without CRS according to the otolaryngologist and the questionnaire.

\begin{tabular}{|c|c|c|c|c|}
\hline & & \multicolumn{2}{|c|}{ Clinical based CRS } & \multirow[t]{2}{*}{ Total } \\
\hline & & Yes & No & \\
\hline $\begin{array}{l}\text { Ques- } \\
\text { tion- }\end{array}$ & Yes & $\begin{array}{c}45(34=76 \% \text { with } \\
\text { asthma or allergic } \\
\text { rhinitis })\end{array}$ & $\begin{array}{c}19 *(16=84 \% \text { with } \\
\text { asthma or allergic } \\
\text { rhinitis })\end{array}$ & 64 \\
\hline $\begin{array}{c}\text { based } \\
\text { CRS }\end{array}$ & No & $\begin{array}{c}46(33=72 \% \text { with } \\
\text { asthma or allergic } \\
\text { rhinitis })\end{array}$ & $\begin{array}{l}252(126=50 \% \\
\text { with asthma or } \\
\text { allergic rhinitis) }\end{array}$ & 298 \\
\hline Total & & 91 & 271 & 362 \\
\hline
\end{tabular}

*According to the otolaryngologist these persons were diagnosed as: Normal (=7), chronic rhinitis $(=7)$, common cold $(=5)$, septal deviation $(=3)$. 
nosed according to the questionnaire but not by the otolaryngologist; either they had no objective findings or they did not fulfil the symptom criteria when interviewed by the otolaryngologist. The mean SNOT 22 total sum score in this group was 22.4, and most were, according to the otolaryngologist, diagnosed as normal followed by chronic rhinitis, common cold, and septal deviation. Only diagnosis related to the nose or sinuses were evaluated. In 46 cases, CRS was diagnosed according to the otolaryngologist but not by the questionnaire. The mean SNOT 22 total sum score in this group was 25.6. Allergic rhinitis and asthma were not diagnosed by the otolaryngologist but was only defined by questionnaire. The percentage of asthma and allergic rhinitis in each group is noted.

According to the otolaryngologist, only 1 person had no symptoms and no objective findings but was diagnosed with CRS because the person was known with CRS and was in medical treatment.

Comparing CRS diagnosed by questionnaire to CRS diagnosed by the otolaryngologist showed moderate agreement (kappa = 47.08).

Comparing clinical-based CRS with EMD-CRS instead of the original questionnaire increased the agreement by raising the kappa value from 47.08 to 57.65. Number of persons with EMDCRS was 77 and only 64 of these persons had CRS according to the original questionnaire. Therefore, 13 patients reported too few subjective symptoms to be diagnosed with CRS based on symptoms only, but were diagnosed with EMD-CRS because of having self reported CRS and being in relevant medical treatment. Sensitivity, specificity, positive predictive values (PPV), and negative predictive values (NPV) adjusted for sex, age, smoking, allergic rhinitis, and asthma was calculated comparing the original questionnaire-based CRS and EMD-CRS with the clinical-based CRS (Appendix 1 and 2). Figure 1 is an extract of Appendix 1 and 2 and includes only the group of persons with no asthma and no allergic rhinitis. Figure 1 illustrates the difference between the original questionnaire-based CRS (row I) and EMD-CRS (row II) by comparing them to the clinical-based CRS. It shows that NPV, but especially sensitivity and PPV, were raised using the EMD-CRS instead of the original questionnaire. The original questionnaire was unable to discriminate between the true positives and false negatives and the associated sensitivities were consequently estimated to 0.5 independently of all covariates. Similarly the EMD-CRS-based sensitivity was estimated to 0.5 when only sex and age were taken into account. However, when additional covariates were included the EMD-CRS-based sensitivity appeared at high levels in persons with no asthma and no allergic rhinitis as shown in Figure 1. A high sensitivity was also found in never-smokers. These high sensitivities implicate that in persons with no asthma and no allergic rhinitis and in never smokers the EMD-CRS diagnosis could identify

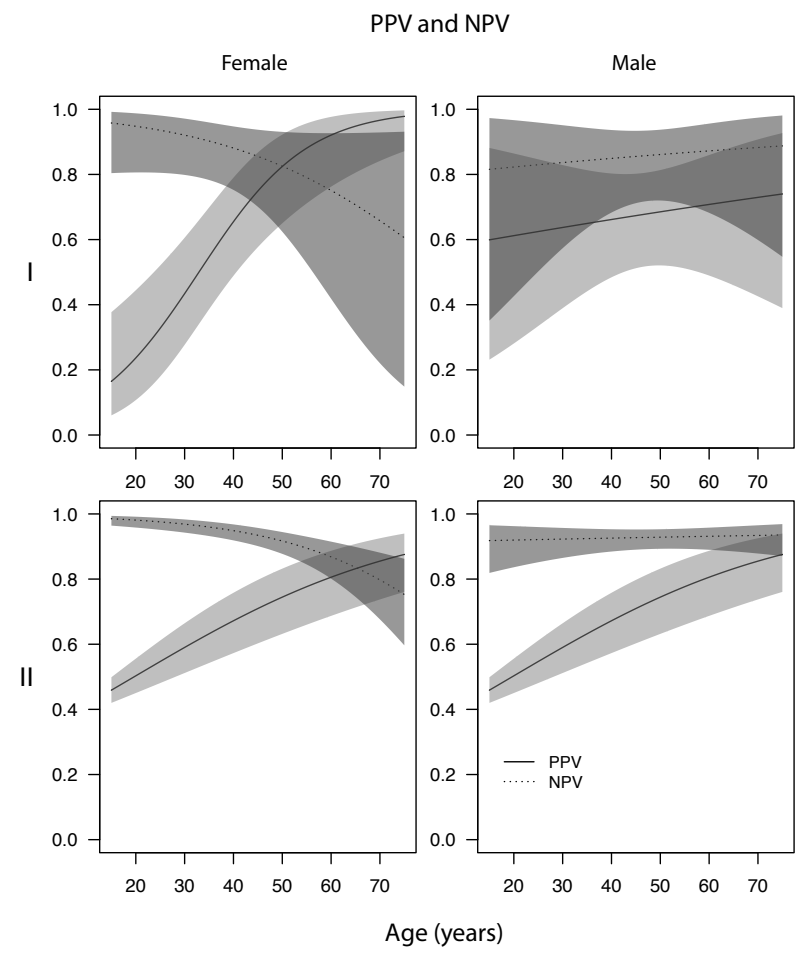

Figure 2. Title. PPV and NPV with 95\% confidence bands comparing questionnaire-based CRS with clinical-based CRS.

Row I: All CRS patients stratified for age and sex. Row II: Patients with no asthma and no allergic rhinitis.

the majority of CRS patients correctly. Figure 2 compares the total group of persons to those with no asthma and no allergic rhinitis when using the original questionnaire. It illustrates that PPV and NPV was better in the group of persons with no asthma and no allergic rhinitis.

For both the original questionnaire and the EMD-CRS the specificity was high and showed age-dependency but with varying trends depending on other covariates. In particular women at $40+$ showed a high level of specificity in all cases when using the EMD-CRS. Both sexes showed PPV increasing with age and women attained acceptable levels above age 40. For EMD-diagnosed men NPV showed a constant high level.

We analyzed how the knowledge of the patients' medical history by the otolaryngologist influenced the agreement between the clinical-based CRS and questionnaire-based CRS. By including or excluding doctor diagnosed CRS and medical treatment in the clinical-based diagnosis the highest agreement was achieved by including the medical history in the clinical diagnosis.

\section{Discussion}

Phenotyping CRS is still an ongoing subject for discussion and CRS is a difficult disease to diagnose as long as we do not have 
biomarkers or other laboratory tests to rely on ${ }^{(12)}$. We are forced to diagnose CRS through the patients variety of symptoms, duration of disease, and, if possible, objective findings. The heterogeneity of the disorder and the diagnostic imprecision is indeed a problem.

The $\mathrm{EP}^{3} \mathrm{OS}$ criteria from 2007 were for the first time used in a trans-European survey, the GA2LEN Survey. The Danish national data from the questionnaire at follow-up was used together with data from the clinical consultation to evaluate if questionnaire-based CRS was reliable when compared to examination by an otolaryngologist.

The golden standard for diagnosing CRS is by an otolaryngologist in an ENT setting. Our setup at the follow-up was as close to an ENT setting as possible. The agreement between CRS based on the questionnaire compared to the otolaryngologist was moderate. The questionnaire was completed at the same occasion as the examination by the otolaryngologist and the difference in agreement can thereby not be explained by seasonal variation or fluctuation in symptoms because of a time interval.

It was evaluated what diagnoses were given by the otolaryngologist to those who had questionnaire-based CRS but not CRS according to the otolaryngologist. Most of these patients were diagnosed as healthy meaning that although people have symptoms according to a questionnaire, some have so few symptoms and/or objective findings that a diagnosis could not be justified. Often a continuum of symptoms is seen and the diagnostic criteria form a cut of value separating patients with mild abnormalities from those needing professional attention and treatment. Diseases like allergic rhinitis, chronic rhinitis, septal deviation, and common cold cause symptoms resembling those of CRS. When dealing with patients having nasal symptoms, these diseases have to be kept in mind, and to differentiate between them and CRS an objective examination of the nose has to be performed as an accurate diagnosis cannot be made otherwise ${ }^{(13)}$. Furthermore, our results showed that in patients with asthma or allergic rhinitis the symptom-based CRS diagnosis is difficult to rely on (Figure 2), and a thorough ENT examination is important among these patients.

As our study was population-based it was expected that the severity of disease was lower than would have been seen in patients selected from a hospital. Indeed our average SNOT22 total sum score was lower when compared to the study by Abdalla et al., where they investigated a group of CRS patients waiting for surgery and having an average score at $44.4^{(14)}$. Comparing clinical-based CRS with the original questionnaire and the questionnaire with the new option (EMD-CRS), we found that the agreement was higher with the new option which includes knowledge of previous doctor diagnosed CRS and treatment. The expected specificity, sensitivity, PPV, and
NPV support this conclusion and in particular they suggest good agreement among never smokers and age 40+. To some extent there was more agreement among female than male participants. It may be that men were less aware of their nasal symptoms and that they did not focus on the specific symptoms or for how long the symptoms had been present. A gender difference in symptom evaluation has previously been described ${ }^{(15)}$

The disagreement between clinical-based CRS and questionnaire-based CRS cannot be explained by asthma, nasal allergy, or difference in the SNOT-22 total sum score as they were all insignificant. The most important factor was the result of nasal endoscopy and control of disease but also a person's symptomawareness changed in some persons between completing the questionnaire and the interview by the otolaryngologist. Incorporation of EMD-CRS should be considered when preparing new questionnaires on CRS and revision of the EPOS criteria. The questionnaire in its original form overlooks the patients who are known with CRS and are in medical treatment resulting in having almost no subjective symptoms (clinical control). We have used and compared the epidemiological and clinical definitions on CRS according to the $\mathrm{EP}^{3} \mathrm{OS}$ criteria. These are useful definitions having their own rights in epidemiology studies and clinical settings respectively.

In this study we compared questionnaire-based CRS to what must be the golden standard, a complete examination by an otolaryngologist in an ENT setting. The results showed that there is still room for adjusting the epidemiological instruments before we can be sure to diagnose the same disease in epidemiology and clinical studies.

\section{Acknowledgement}

The study was supported by the European Commission as part of the FP6 Program through the allocation made to the GA²LEN network and the Integration Research Work Package.

\section{Authorship contribution}

Co-authorships are assigned according to the Vancouver guidelines.

\section{Conflict of interest}

The study was part of a Ph.D. study, which was financial supported by: the department of Otorhinolaryngology, Odense University Hospital, the University of Southern Denmark, the Region of Southern Denmark, Schering Plough, Poul Traun-Pedersen's memorial fund, and Hans Skouby's fund. The supporting organisations had no part in the protocol or conductance of the study and had no financial interest in the outcome. 


\section{References}

1. Hastan D, Fokkens WJ, Bachert C, Newson RB, Bislimovska J, Bockelbrink A, et al. Chronic rhinosinusitis in Europe - an underestimated disease. A GA(2)LEN study. Allergy. 2011; 66: 1216-1223.

2. Thilsing T, Rasmussen J, Lange B, Kjeldsen AD, Al-kalemji A, Baelum J. Chronic rhinosinusitis and occupational risk factors among 20- to 75-year-old Danes-A GA(2)LEN-based study. Am J Ind Med. 2012; 55: 1037-1043.

3. Fokkens WJ, Lund VJ, Mullol J. European position paper on rhinosinusitis and nasal polyps 2007. Rhinology. 2007; Suppl 20: 1-136.

4. Stankiewicz JA, Chow JM. Nasal endoscopy and the definition and diagnosis of chronic rhinosinusitis. Otolaryngol Head Neck Surg. 2002; 126: 623-627.

5. Holbrook EH, Brown CL, Lyden ER, Leopold DA. Lack of significant correlation between rhinosinusitis symptoms and specific regions of sinus computer tomography scans. Am J Rhinol. 2005; 19: 382-387.

6. Tahamiler R, Canakcioglu S, Ogreden S, Acioglu E. The accuracy of symptom-based definition of chronic rhinosinusitis. Allergy. 2007; 62: 1029-1032.

7. Tomassen P, Newson RB, Hoffmans R,
Lotvall J, Cardell LO, Gunnbjornsdottir M, et al. Reliability of EP3OS symptom criteria and nasal endoscopy in the assessment of chronic rhinosinusitis - a GA(2)LEN study. Allergy. 2011; 66: 556-561.

8. Jarvis D, Newson R, Lotvall J, Hastan D, Tomassen P, Keil T, et al. Asthma in adults and its association with chronic rhinosinusitis: The GA(2) LEN survey in Europe. Allergy. 2012; 67: 91-98.

9. Lange B, Thilsing T, Al-kalemji A, Baelum J, Martinussen T, Kjeldsen A. The Sino-Nasal Outcome Test 22 validated for Danish patients. Dan Med Bull. 2011; 58: A4235.

10. Agresti A. Categorical data analysis. 2nd ed ed. New York: Wiley; 2002

11. Kirkwood BR, Sterne JAC. Essential medical statistics. 2. edition ed. Malden, Mass. Blackwell Science; 2003

12. Bachert C, Van BN, Toskala E, Zhang N, Olze $H$, Scadding $G$, et al. Important research questions in allergy and related diseases: 3-chronic rhinosinusitis and nasal polyposis - a GALEN study. Allergy. 2009; 64: 520-533.

13. Bousquet J, Fokkens W, Burney $P$, Durham SR, Bachert C, Akdis CA, et al. Important research questions in allergy and related diseases: nonallergic rhinitis: a GA2LEN paper. Allergy. 2008; 63: 842-853.
14. Abdalla S, Alreefy H, Hopkins C. Prevalence of sinonasal outcome test (SNOT-22) symptoms in patients undergoing surgery for chronic rhinosinusitis in the England and Wales National prospective audit. Clin Otolaryngol. 2012; 37: 276-282.

15. Barsky AJ, Peekna HM, Borus JF. Somatic symptom reporting in women and men. J Gen Intern Med. 2001; 16: 266-275.

\section{Bibi Lange}

Department of Otorhinolaryngology

Odense University Hospital

Sdr. Boulevard 29

DK-5000 Odense $C$

Denmark

Tel: $+45-65412810$

E-mail: Bibi.lange@ouh.re-

gionsyddanmark.dk 
Appendix 1. Title. Sensitivities, specificities, positive predictive values, and negative predictive values comparing questionnaire-based CRS with clinical-based CRS.

Model based sensitivities, specificities, positive predictive values, and negative predictive values with $95 \%$ confidence bands at age for males and females comparing questionnaire-based and clinical-based CRS. In the perfect situation all bands would be situated in the top of each figure for all aged which they are not. Instead there are broad confidence intervals among the very young age groups and among the older age groups.

Row I: only stratified for age and sex. Row II: No Asthma, No Allergic rhinitis. Row III: Asthma, No Allergic rhinitis. Row IV: No Asthma, Allergic rhinitis. Row V: Asthma and Allergic rhinitis.

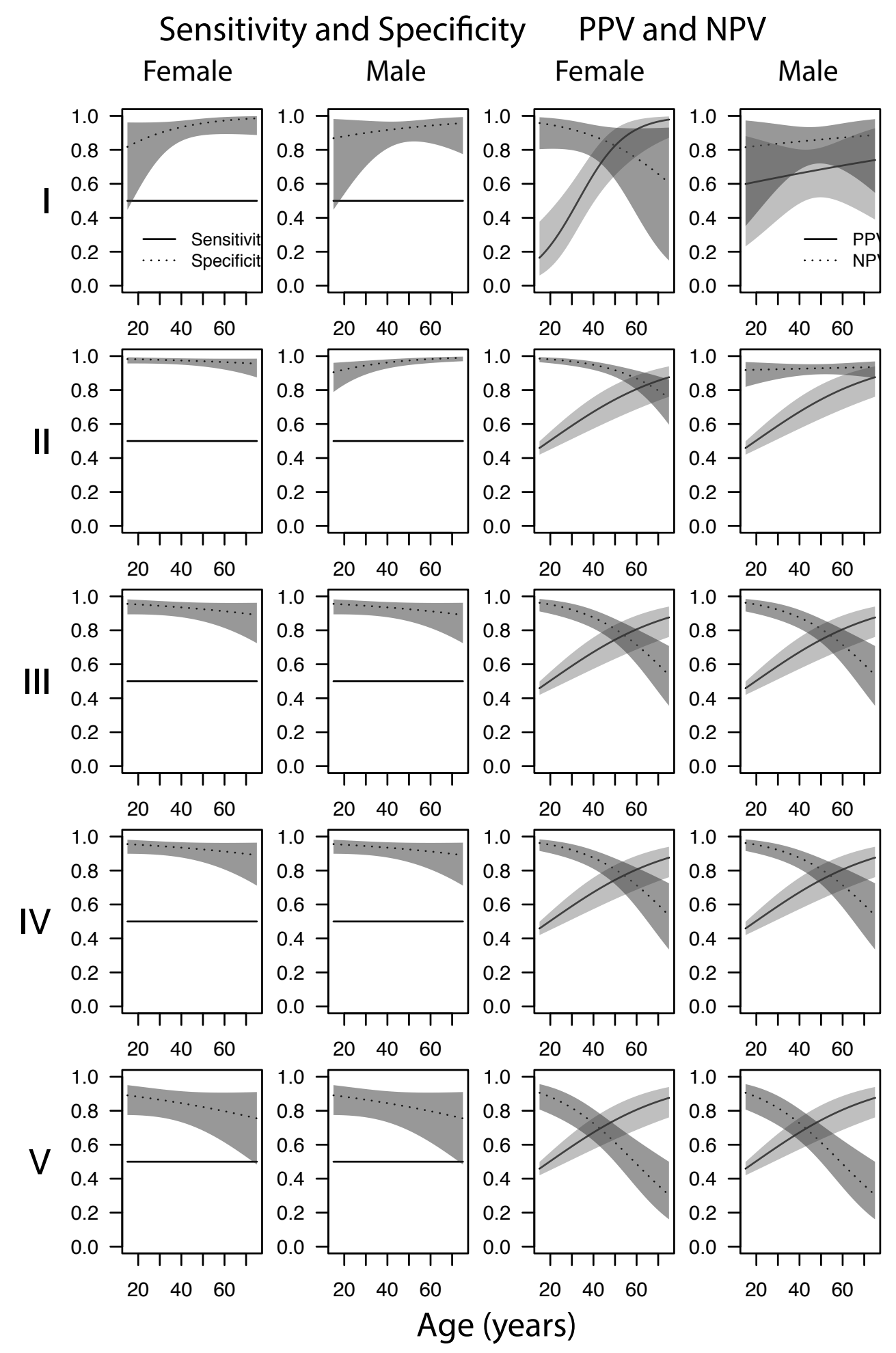


Appendix 2. Title: Sensitivities, specificities, positive predictive values, and negative predictive values comparing EMD-CRS with clinical-based CRS. Model based sensitivities, specificities, positive predictive values, and negative predictive values with $95 \%$ confidence bands at age for males and females comparing EMD-CRS and clinical-based CRS. In the perfect situation all bands would be situated in the top of each figure for all aged which they are not. Instead there are broad confidence intervals among the very young age groups and among the older age groups.

Row I: Only stratified for age and sex. Row II: No Asthma, No Allergic rhinitis, Never Smoker. Row III: No Asthma, No Allergic rhinitis, Ex-Smoker. Row IV: No Asthma, No Allergic rhinitis, Smoker. Row V: Asthma, No Allergic rhinitis, Never Smoker. Row Vl: No Asthma, Allergic rhinitis, Never Smoker.

\section{Sensitivity and Specificity PPV and NPV}

Female

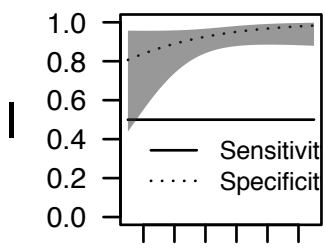

204060

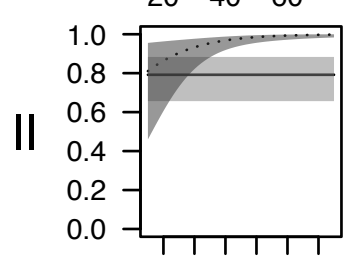

204060

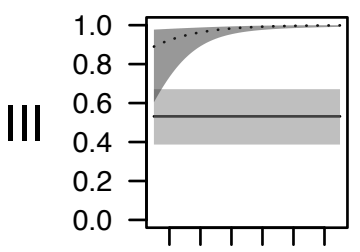

$20 \quad 40 \quad 60$
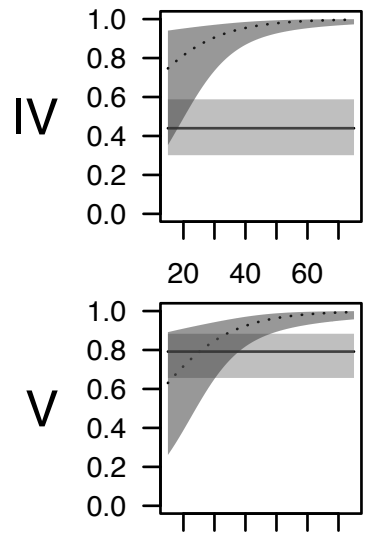

$20 \quad 40 \quad 60$

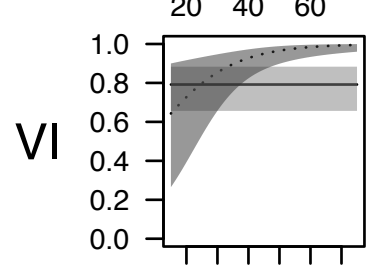

$20 \quad 40 \quad 60$
Male

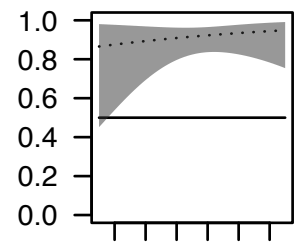

$20 \quad 40 \quad 60$

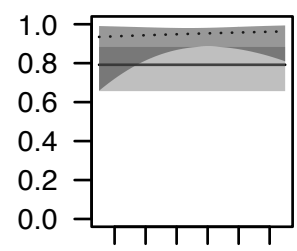

$20 \quad 40 \quad 60$

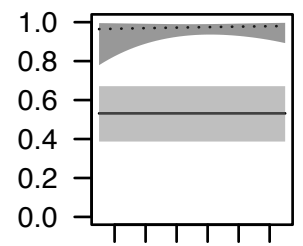

$20 \quad 40 \quad 60$

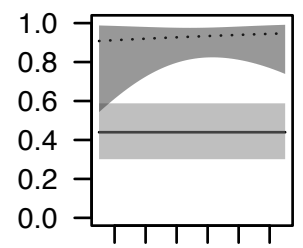

$20 \quad 40 \quad 60$

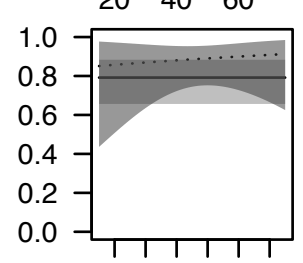

$20 \quad 40 \quad 60$

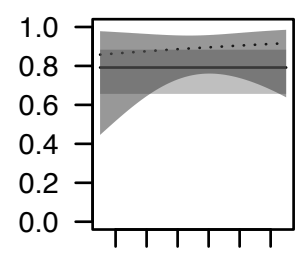

$20 \quad 40 \quad 60$
Female

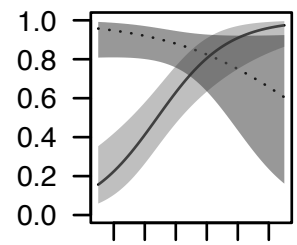

$20 \quad 40 \quad 60$

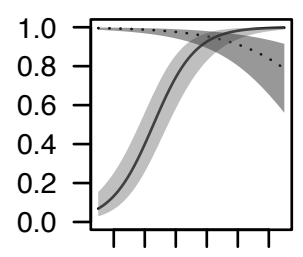

$20 \quad 40 \quad 60$

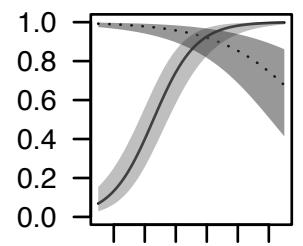

$20 \quad 40 \quad 60$

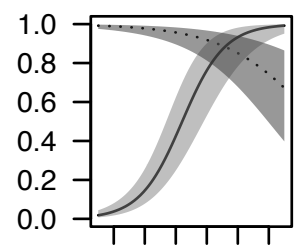

$20 \quad 40 \quad 60$

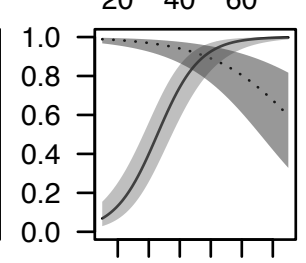

$20 \quad 40 \quad 60$

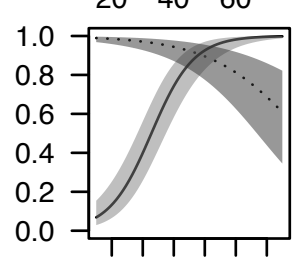

$20 \quad 40 \quad 60$

Age (years)
Male

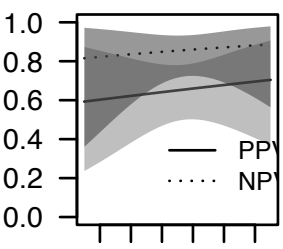

$20 \quad 40 \quad 60$

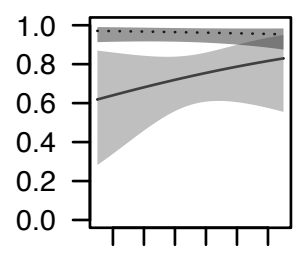

$20 \quad 40 \quad 60$

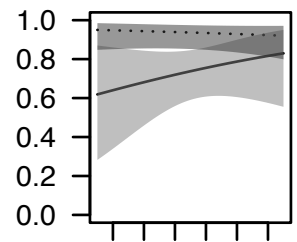

$20 \quad 40 \quad 60$

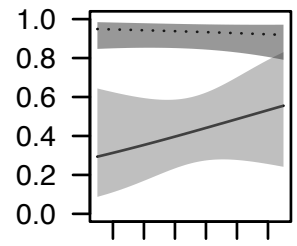

$20 \quad 40 \quad 60$

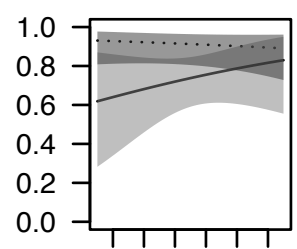

$20 \quad 40 \quad 60$

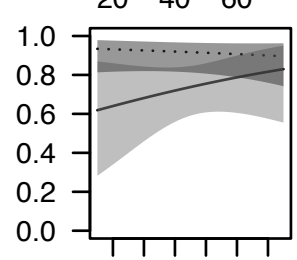

$20 \quad 40 \quad 60$ 